\title{
An integral role for timing in interception
}

Chia-Jung Chang ${ }^{1}$ and Mehrdad Jazayeri ${ }^{1,2}$

${ }^{1}$ Department of Brain and Cognitive Science, ${ }^{2}$ McGovern Institute for Brain Research, Massachusetts Institute of Technology, Cambridge, Massachusetts 02139, USA.

Correspondance should be addressed to M.J. (mjaz@mit.edu)

Mehrdad Jazayeri, PhD

Robert A. Swanson Career Development Professor

Assistant Professor, Dept. of Brain and Cognitive Sciences

Investigator, McGovern Institute for Brain Research

43 Vassar Street, Building 46-6041,Cambridge, MA 02139-4307

Massachusetts Institute of Technology

Phone: $617-715-5418$

Email:mjaz@mit.edu

Key Words: object interception, cue combination, speed estimate, temporal statistics, Bayesian model

\section{$\underline{\text { Acknowledgements }}$}

We thank Rossana Chung and Feitong Yang for comments on the manuscript. M.J. is supported by NIH (NINDS-NS078127), the Sloan Foundation, the Klingenstein Foundation, the Simons Foundation, the McKnight Foundation, the Center for Sensorimotor Neural Engineering, and the McGovern Institute.

\section{$\underline{\text { Author Contribution }}$}

C.-J.C. designed the experiment, collected and analyzed the data, and performed the computational modeling. M.J. helped in experimental design, data analysis, and provided intellectual support throuout the study. C.-J.C. and M.J. wrote the manuscript. 


\section{Abstract}

1 Timing is critical for myriad behaviors in dynamic environments. For example, to intercept an object, the

2 brain must compute a reliable estimate of time-to-contact (TTC). Prior work suggests that humans

3 compute TTC using kinematic information such as distance and speed without explicitly relying on

4 temporal cues, just as one would do in a physics classroom using kinematic equations. Considering the

5 inherent uncertainty associated with estimates of speed and distance and the ability of human brain to

6 combine different sources of information, we asked whether humans additionally rely on temporal cues.

7 We found that humans actively integrate speed information with both explicit and implicit timing cues.

8 Analysis of behavior in relation to a Bayesian model revealed that the additional temporal information

9 helps subjects optimize their performance in the presence of measurement uncertainty. These findings

10 suggest that brain's timing mechanisms are actively engaged while interacting with dynamic stimuli. 


\section{Introduction}

11 Imagine intercepting a moving ball on a pool table as it bounces around hitting different edges. Does one

12 only process kinematic information such as distance, position and speed, or does one additionally pay

13 attention to when the ball hits the edges? At first glance, the answer seems trivial: since kinematic

14 variables and time are directly related through kinematic equations (e.g., $\mathrm{t}=\mathrm{d} / \mathrm{v}$ ), there would be no

15 additional advantage in tracking time. What if internal estimates of speed and position were unreliable,

16 for example, the lights were too dim to clearly see the ball? In that case, one may choose to pay attention

17 to when the ball hits the edges to improve accuracy. This example highlights a general, important, and

18 unresolved question in sensorimotor processing: do humans actively engage brain's timing mechanisms

19 when interacting with dynamic stimuli, or do they solely rely on kinematic information?

20 We aimed to address this question using a virtual object interception task. When intercepting a moving

21 object, one has to estimate when the object reaches a desired target location, a variable that we will refer

22 to as time-to-contact (TTC). Early studies hypothesized that TTC is derived from the rate of expansion of

23 an object's retinal image ${ }^{1-3}$. Later, it was suggested that TTC is derived indirectly from an object's speed

24 and position ${ }^{4-13}$. For example, when an object moves with a fixed speed, TTC would be computed by

25 dividing perceived distance by perceived speed. However, as the example of the pool table illustrates, the

26 inherent variability in the measurement and processing of kinematic information ${ }^{14-20}$ renders temporal

27 cues highly relevant for the estimation of TTC. Humans' ability to measure time intervals independent of

28 kinematic cues is well-documented ${ }^{21-24}$. Furthermore, decades of research indicate that humans can

29 efficiently combine multiple sources of information ${ }^{14,25-32}$. Therefore, we hypothesized that humans

30 integrate kinematic information with explicit and implicit temporal cues to derive better estimates of TTC

\section{1 (Box 1).}


32 To test this hypothesis, we designed a series of experiments in which the subjects had to press a key when

33 a bar moving along a linear path would arrive at a target position (Fig. 1a). While moving, the bar was

34 sometimes visible and sometimes occluded. By varying the temporal structure between visible and

35 occluded part of the path, we manipulated the reliability of the speed and the temporal information

36 independently to compute TTC. Consistent with our hypothesis, we found that subjects actively used

37 temporal information to improve their estimates of TTC.

38 To better understand the nature of the underlying computations, we compared subjects' behavior to that of

39 an ideal Bayesian observer who optimally integrates speed and timing information. Similar to work in

40 other sensorimotor domains ${ }^{14,25-32}$, the model was able to accurately capture subjects' estimation strategy

41 indicating that humans efficiently integrate prior statistics with measurements of both speed and elapsed

42 time. These results highlight a hitherto unappreciated function of the brain's capacity to utilize time -

43 independent of speed and distance - to inform sensorimotor function while interacting with dynamic

44 stimuli.

\section{Results}

\section{Experiment 1: Interception performance benefits from explicit timing cues}

45 We first asked whether humans are capable of integrating speed information with temporal cues to

46 improve their estimate of TTC in an interception task. To do so, we asked subjects to intercept a moving

47 bar in three conditions: one with a speed cue, one with a timing cue, and one with both cues present (Fig.

48 2a). In the first condition, the bar was visible only in the early part of the path, and then was occluded

49 before it reached the target position. Subjects had to measure the speed of the bar from an early visible

50 segment and use that to compute when the bar would reach the target position at the end of the occluded

51 segment. We denote this condition by $\mathrm{I}(\mathrm{VM})$ as a shorthand for Interception in the presence of Visible

52 Motion. 
53 In the second condition, the motion was invisible but the position of the bar was flashed at the beginning

54 of the path and when it reached the central fixation point, which was halfway along the path. TTC had to

55 be computed based on the interval between two flashes along the path. We placed the timing cue around

56 the fixation point to avoid causing a gaze shift in response to the flash, which could impact the subjects'

57 overall estimation strategy. We denote this condition by I(FP) as a shorthand for Interception in the

58 presence of Flashed Position.

59 In the third condition, the bar was visible early on, and additionally, its position was flashed briefly at

60 central fixation point, i.e., halfway between the initial and target location, giving subjects the opportunity

61 to measure both speed and timing information. We denote this condition by $\mathrm{I}(\mathrm{VM}+\mathrm{FP})$.

62 The actual time-to-contact (TTCa) was defined as the time from when the bar passed the central fixation

63 to when it reached the target. We compared TTCa to the interval between when the bar passed the central

64 fixation and subjects pressed the button. We refer to this interval as the produced time-to-contact (TTCP).

65 For I(FP) and I(VM+FP) conditions, TTCp was straightforwardly computed from the time when the bar

66 was flashed to when the button was pressed. For the I(VM) condition, because the bar was not visible

67 throughout the path (not flashed at the fixation point), we estimated TTCP by appropriately scaling the

68 response time by the occluded segment.

69 As evident from the $T T C p$ pattern for a typical subject (Fig. 2a), subjects were able to perform the task in

70 all three conditions with different degrees of of sensitivity. TTC $p$ values were variable and systematically

71 biased toward the mean. We quantified this regression to the mean by computing a BIAS term that

72 quantifies the overall deviation from the identity line (see Methods; Fig. 2b). The BIAS was significantly

73 smaller when both cues were available compared to the speed cue condition $\left(\mathrm{t}_{198}=26.6435, \mathrm{p}<0.001\right.$,

74 hedges' $g=3.7537)$ as well to the timing cue condition $\left(\mathrm{t}_{198}=27.4602, \mathrm{p}<0.001\right.$, hedges' $\left.\mathrm{g}=3.8687\right)$.

75 This reduction in BIAS was observed for all the subjects (Fig. 2c) and was significant across subjects 
76 (Wilcoxon one-side signed-rank test, statistics $=28, \mathrm{p}<0.01$ ), suggesting that humans are capable of

77 integrating speed information with temporal cues to reduce uncertainty.

78 While this result is consistent with subjects integrating the two cues, it is also possible that the subjects

79 did not integrate the two cues and instead used the timing cue (flash at the fixation point) to simply reset

80 their subjective estimate of the position of the bar to the central fixation. To test this possibility, we tested

81 a subset of subjects in a cue conflict paradigm in which the flash at the central fixation (FP) was jittered

82 by -100 , 0, or 100 msec relative to when the bar reached the central fixation (Supplementary Fig. 1a).

83 To understand the logic of this experiment, let us predict subjects' estimated time-to-contact (TTCe)

84 under various hypotheses for a case where the flash is presented $100 \mathrm{~ms}$ later that when the bar reaches

85 the fixation point. If a subject only relies on the timing cue, they would overestimate the time from

86 motion onset to the flash by $100 \mathrm{msec}$; this would predict that TTCe would be $100 \mathrm{msec}$ longer than

$87 T T C a$. If a subject only relies on speed, then the lagging flash would not influence the subject's behavior.

88 However, since we quantify $T T C p$ with respect to the time when the bar is flashed, we would register a

89 TTCe that would be 100 shorter than TTCa. As a third hypothesis, let us consider that a subject would use

90 the flash to reset the position, and use the speed to estimate TTCe. For this hypothesis, the TTCe would

91 remain the same as when the subject only used the speed cue. Finally, if the subjects were to integrate the

92 two cues, we would see a bias in TTCe in the direction of the jitter that would be less than 100 msec away

93 from the non-jittered condition. The results were consistent with the last hypothesis of integration and

94 could not be explained by hypotheses including the position-reset hypothesis (Supplementary Fig. 1a).

\section{Experiment 2: Interception performance benefits from inherent timing cues}

95 Experiment 1 demonstrated that humans were able to integrate timing cue with speed information.

96 However, this could have been due to the presentation of an explicit timing cue (i.e., the flash a the

97 fixation point). Therefore, we asked whether subjects utilize the timing information from the visible

98 portion of the motion even if no explicit flash at the fixation point is presented. To validate the role of 
99 time as an additional cue, it was important to make sure that longer visible motion did not additionally

100 improve subjects' estimate of the speed. Therefore, as a first step, we measured the ability of subjects to

101 estimate speed during interception with different visible lengths ranging from 0.625 to 5 degree in log

102 scale while keeping the occluded distance fixed (Supplementary Fig. 2). We evaluated performance by

103 measuring subjects' root mean squared error (RMSE). We found that performance improved significantly

104 as the visible lengths increased from 0.625 to 1.25 degree (paired-sample t-test, $\mathrm{t}_{399}=56.61, \mathrm{p}<0.001$ )

105 and saturated afterwards (paired-sample t-test, $\mathrm{t}_{399}=0.9031, \mathrm{p}=0.3670$ ). In other words, the fidelity of

106 the speed estimate saturated at a visible length of 1.25 degree.

107 We then conducted an interception task where we evaluated the relevance of the timing cue by changing

108 the visible length compared to the occluded length. Importantly, in all conditions, the visible length was

109 beyond the saturation point in the I(VM) task. This ensured that any improvement in performance was not

110 due to an improvement of speed estimates. We tested subjects' performance in three conditions. In all

111 conditions, the occluded length was fixed $\left(\mathrm{d}_{2}=8\right.$ degree). Across conditions, the ratio of the occluded

112 length $\left(d_{2}\right)$ to the visible length $\left(d_{1}\right)$ was varied by a gain factor $\left(G=d_{2} / d_{1}\right)$. The three gain factors were

$113 \quad 0.667,1$, and 1.6.

114 Figure 3a and $\mathbf{3 b}$ shows the performance of a typical subject in the three conditions. Surprisingly, the

115 best performance was not associated with $\mathrm{G}=0.667$ when the visible length was longest. Instead, RMSE

116 was smallest when the visible and occluded lengths were equal $\left(\mathrm{G}=0.667, \mathrm{t}_{198}=20.3981, \mathrm{p}<0.001\right.$,

117 hedges' $\mathrm{g}=2.9308 ; \mathrm{G}=1.6, \mathrm{t}_{198}=22.9261, \mathrm{p}<0.001$, hedges' $\mathrm{g}=3.2299$ ), which we refer to as the

118 identity condition. The same was true across subjects (Fig. 3c; Wilcoxon one-side signed-rank test,

119 statistics $=28, \mathrm{p}<0.01)$ revealing a systematic and consistent improvement of performance in the identity

120 condition. 
121 The same group of subjects were also tested in the I(FP) task, and for the same three gains. As evident

122 from the behavior of the same typical subject, RMSE was smaller when the measurement and production

123 intervals were the same (Fig. 3d and 3e) compared to when the measurement period was longer $(\mathrm{G}=$

$1240.667, \mathrm{t}_{198}=29.7316, \mathrm{p}<0.001$, hedges' $\left.\mathrm{g}=4.1887\right)$, or shorter $\left(\mathrm{G}=1.6, \mathrm{t}_{198}=25.6390, \mathrm{p}<0.001\right.$,

125 hedges' $g=3.6122$ ). This effect was present across subjects (Fig. 3f; Wilcoxon one-side signed-rank test,

126 statistics $=28, \mathrm{p}<0.01)$ indicating that spatiotemporal identity helped subjects improve their estimate of

127 TTC. We also compared subjects' performance in the identity condition between the I(FP) and I(VM)

128 conditions (Supplementary Fig. 3a). RMSE was consistently and significantly smaller in the I(VM)

129 condition (Wilcoxon one-side signed-rank test, statistics $=3, \mathrm{p}<0.001$ ). This ruled out the possibility that

130 subjects switched to a pure timing strategy in the identity context. These results suggest that subjects

131 exploited the temporal structure to improve their performance.

\section{Experiment 3: Interception performance improves with temporal-identity context}

132 Experiment 2 clearly demonstrated that interception was most accurate in the identity context when the

133 visible and occluded parts of the path were identical. This is consistent with our hypothesis that

134 performance benefited from the fact that the visible and occluded intervals had the same duration (i.e.,

135 temporal identity) allowing subjects to more accurately estimate TTC. However, it is also possible that

136 this improvement was because the visible and occluded parts had the same length (i.e., distance identity)

137 allowing subjects to better estimate distance. The latter hypothesis seems unlikely given that the occluded

138 distance was fixed throughout all experiments. Nonetheless, we conducted an additional experiment to

139 assess the relevance of temporal versus distance identity in interception performance.

140 Since distance and duration are related through speed, the only way to dissociate the two is to make the

141 speed of the bar differ between the visible and occluded parts of the path. Therefore, we designed a

142 variant of the interception task in which, unbeknownst to the subjects, the speed of the bar behind the

143 occluder was made 1.25 times faster than the speed in the visible portion (Fig. 4a). The non-identical 
144 speed ratio enables us to create conditions in which the distance and temporal identity were dissociated.

145 In one condition, the visible and occluded distances were the same creating distance identity $\left(\mathrm{G}_{\mathrm{d}}=1\right)$

146 without temporal identity $\left(\mathrm{G}_{\mathrm{t}}=1 / 1.25\right)$. In another condition, we matched the ratio of the distances to the

147 ratio of the speeds $\left(G_{d}=1.25\right)$ to create temporal identity $\left(G_{t}=1\right)$.

148 A new set of subjects was recruited for this experiment to ensure that any sensitivity to temporal context

149 was not because of participation in previous experiments. Since subjects were not aware of the speed

150 change behind the occluder, they could only adjust their performance based on feedback. We compared

151 subjects' performance between the $G_{d}=1$ and $G_{t}=1$ conditions. We reasoned that an observer that relies

152 on the distance identity should have higher performance (lower RMSE) in the $G_{d}=1$ condition. In

153 contrast, an observer that relies on the temporal identity would have a lower RMSE in the $\mathrm{G}_{\mathrm{t}}=1$ despite

154 the fact that the distances between the visible and occluded parts are not the same.

155 We found that RMSE was lower for the temporal identity compared to distance identity condition as

156 shown for a typical subject (Fig. $4 \mathbf{b}$ and $4 \mathbf{c} ; \mathrm{t}_{198}=25.6431, \mathrm{p}<0.001$, hedges' $\mathrm{g}=3.6127$ ) and across

157 subjects (Wilcoxon one-side signed-rank test, statistics $=34, \mathrm{p}<0.05$ ). This finding further substantiates

158 our conclusion that subjects rely more on temporal context to estimate TTC.

\section{Bayesian integration of speed and time explains interception performance}

159 Experiments 1 to 3 established that subjects integrate speed and timing information to improve their

160 performance. Another salient feature of subjects' behavior across all conditions (Fig. 2 and Fig. 3 and

161 Fig. 4), was that TTCP was biased toward the mean of the prior. This was true for the external timing cue

162 tasks in Experiment 1, for the inherent timing tasks in Experiment 2, and in the control condition in

163 Experiment 3, regardless of the ratio of the occluded to visible lengths (different values of G). Together,

164 these observations suggest that subjects performance may be explained by a Bayesian model that

165 integrates the prior with both the speed and timing information (Fig. 5a). 
166 To test this hypothesis rigorously, we developed an ideal Bayesian observer for the task. We assumed that

167 the ideal observer made two conditionally independent measurements while the moving bar was visible,

168 one associated with the speed of the bar $\left(v_{m}\right)$, and another associated with the duration of the visible

169 interval $\left(t_{m}\right)$. Following previous work, we assumed that these measurement were subject to scalar

170 variability ${ }^{26,33-36}$. In particular, we assumed that the standard deviation of noise on speed scaled with the

171 bar's speed $(v)$ with constant of proportionality $\left(w_{m V}\right)$ and standard deviation of noise on elapsed time

172 scaled with visible duration $\left(t_{l}\right)$ with constant of proportionality $\left(w_{m T}\right)$. The ideal observer integrated the

173 prior, $p\left(t_{1}\right)$, with the likelihood of the bar speed, $\lambda\left(v_{m} \mid v\right)$ and the likelihood of the visible duration, $\lambda\left(t_{m} \mid t_{1}\right)$,

174 and computed TTCe from the mean of the posterior. Since this observer minimizes the least-squares error,

175 we will refer to this as the Bayes least-squares (BLS) estimator. To compare the model to subjects'

176 behavior, we augmented the ideal observer with a production stage by adding scalar noise with constant

177 of proportionality $\left(w_{p}\right)$ to TTCe to values of TTCp that incorporated motor variability.

178 We first estimated $w_{m V}, w_{m T}$ for each subject. In most Bayesian models, the model is evaluated by

179 assessing the quality of model fits to the data. A more powerful approach is to fit the model to a training

180 dataset and examine how well it explains a test dataset. An even more powerful approach is to fit the

181 model to one set of conditions and ask whether it predicts data in another condition to which it was not

182 fitted. We employed the last approach. For each subject, we estimated $w_{m T}$ from the I(FP) task with $\mathrm{G}=1$

183 (Fig. 5b, left), and $w_{m}$ from $\mathrm{I}(\mathrm{VM}+\mathrm{FP})$ in $\mathrm{G}=0.667$ (Fig. 5b, right), and used those estimates to predict

184 subjects' behavior in the $\mathrm{I}(\mathrm{VM}+\mathrm{FP})$ in $\mathrm{G}=1$ (Fig. 5c).

185 To estimate $w_{m T}$, we developed a Bayesian observer for the $\mathrm{I}(\mathrm{FP})$ task with $\mathrm{G}=1$. In this task, the sensory

186 information provided was the interval between when the bar started to move and when it reached halfway

187 along the path (over the fixation point), which we denote by $t_{l}$. We fitted subjects' behavior by a BLS

188 estimator that only relied on the likelihood of $t_{l}, \lambda\left(t_{m} \mid t_{l}\right)$ and the prior distribution, $p\left(t_{1}\right)$. As shown for one 
189 subject (Fig. 5b, left) and consistent with previous work in a similar task ${ }^{21,37-39}$, the model accurately 190 captured subjects' behavior.

191 Next, we estimated $w_{m V}$ from fits of the Bayesian model to the $\mathrm{I}(\mathrm{VM}+\mathrm{FP})$ task when $\mathrm{G}=0.667$. For this 192 fitting procedure, we used the corresponding $w_{m T}$ from the I(FP) task with $\mathrm{G}=0.667$ (see Methods). As 193 shown for the same subject (Fig. 5b, left), the model successfully accounted for the behavior. Recall that 194 in the I(VM+FP) task, we had made the visible length long enough so that subjects' estimate of speed had 195 saturated and was thus no longer dependent on G (Supplementary Fig. 2). This allowed us to safely use 196 the fit to $w_{m V}$ derived from the $\mathrm{G}=0.667$ condition to predict behavior in the $\mathrm{G}=1$ condition.

197 Finally, we use each subject's fits to $w_{m V}, w_{m T}$ to predict the behavior in I(VM+FP) task when gain is one $198(\mathrm{G}=1)$. The model was able to predict the observed TTCp values as shown for one example subject (Fig. 199 5c) and captured the data's summary statistics (BIAS and VAR) across subjects (Fig. 5d). This is 200 remarkable considering that both $w_{m V}$ and $w_{m T}$ were estimated from other tasks, and provides strong 201 support that subjects integrate prior information, speed information, and timing information to optimize 202 their estimate of TTC.

203 To further evaluate the success of the Bayesian model in explaining how subjects integrate speed and 204 timing information, we tested the model in Experiment 1 where the timing cue was provided explicitly by 205 a flash at the fixation point. To create a predictive model, we used the same procedure as we did to predict 206 behavior in Experiment 2. We estimated $w_{m V}$ from data in $\mathrm{I}(\mathrm{VM})$ condition, and $w_{m T}$ from data in I(FP) 207 condition (Supplementary Fig. 3b), and used those values to predict behavior in I(VM+FP) condition 208 (Supplementary Fig. 1b). Again, the model successfully captured the statistics of subjects' behavior 209 suggesting that the brain is optimized for integrating speed and timing information during interception 210 tasks regardless of how timing information is provided. 


\section{Discussion}

211 Our work builds on a large body of work investigating the computational principles of object interception.

212 Early studies hypothesized that humans rely on variables derived from an object's visual angle and its rate

213 of expansion on the retina, of which the so-called tau is a classic example ${ }^{1-3}$. Later, this proposal was

214 deemed inadequate as it failed to capture many empirical observations $s^{4,9,40-42}$. Most current models are

215 based on the idea that interception relies on measurements of kinematic variables $6,7,11,12,43$, such as

216 speed $^{6,32,44}$, distance and/or depth ${ }^{45}$. This idea has also been used in experiments similar to ours where the

217 object moves behind an occluder ${ }^{6,9,46-48}$. In those cases, it is assumed that humans estimate speed while

218 the object is visible and use that estimate to predict future position of the object behind the occluder. This

219 focus on kinematics is natural as it matches our intuition about the physics of how objects move.

220 However, the algorithms the brain uses for object interception need not match our physics intuition. Here,

221 we asked whether humans solely rely on kinematics (e.g., speed and distance), or do they additionally rely

222 on temporal cues and contexts.

223 Real world object interception involves a decision to initiate a movement followed by online adjustments

224 of the movement based on sensorimotor feedback. Although successful interception requires a tight

225 coordination between the initiation and the subsequent adjustments, the two processes typically involve

226 different computations ${ }^{49}$. The decision of when to initiate is, by and large, determined by a prediction of

227 how long it would take to reach the object - i.e., time-to-contact (TTC), whereas the subsequent

228 adjustment involves fine adjustments after the movement has been initiated. Here, we focused on the

229 former asking how the brain determines TTC. To do so, we designed a virtual interception task in which

230 subjects "intercepted" a moving bar by pressing a button when the bar reached a target position behind an

231 occluder. With this design, we effectively eliminated the need for post-initiation adjustments. Our main

232 objective was to investigate whether TTC was computed solely from estimates of bar kinematics (e.g.,

233 speed and distance), or whether subjects additionally relied on temporal cues and contexts. 
234 We tested this question in two complementary sets of experiments. In the first set, we presented a brief

235 flash showing the position of the bar after its disappearance behind the occluder. This flash provided

236 explicit information about position and time of the bar but not its speed. Therefore, any improvement in

237 performance due to the flash must be taking advantage of timing mechanisms in the brain. Results

238 confirmed that subjects could intercept the bar without any speed information, and when the flash was

239 presented along with additional speed information, subjects were able to integrate the two to improve

240 their performance. This result complements a large body of evidence that humans are able to fuse sensory

241 information from multiple modalities while making perceptual inferences ${ }^{29,31,50}$. Note that the integration

242 of speed and time is distinct from the indirect role that time would play by improving one's estimate of

243 speed $^{51-53}$. As we demonstrated in a supporting experiment (Supplementary Fig. 2), the improvement of

244 speed estimate with time saturates rapidly and cannot account for our finding. What our results reveal is

245 that humans actively use elapsed time as an independent cue and integrate it with other visual cues when

246 interacting with dynamic stimuli.

247 In the second set of experiments, we removed the explicit timing cue and instead asked whether subjects

248 would naturally exploit implicit timing cues present in the temporal structure of the environment. To

249 address this question, we designed an interception task in which we varied the interval the bar was visible.

250 Based on recent work ${ }^{54}$, we reasoned that when the visible and occluded epochs have the same duration,

251 subjects would automatically make use of this temporal identity to improve their performance. Subjects'

252 performance was remarkably improved in the temporal identity context compared to when the durations

253 of the visible and occluded regions were not the same. Indeed, this experiment revealed a surprising

254 aspect of human behavior: performance in the identity context was even better than when the occluded

255 length was the same and the visible length was made longer. In other words, prolonging the visible

256 portion was harmful to performance when it broke the temporal structure conferred by the identity

257 context. This result powerfully demonstrated that the key factor driving the performance improvement

258 was the presence of the identity context. This conclusion was reinforced by control experiments showing 
259 that the result was due to temporal - not distance - identity. Finally, we found that subjects' ability to

260 integrate speed and timing information reached performance levels similar to an ideal Bayesian observer,

261 suggesting that human brain is inherently optimized to combine speed and time information for object 262 interception.

263 These experiments lead to a simple and novel conclusion that humans actively engage timing mechanisms

264 during interception. To put this finding in context, it is important to distinguish between the role of time

265 during the visible and occluded regions of the path. When an object moves behind an occluder, subjects

266 could no longer measure the object's speed and thus have no choice but to rely on their sense of time.

267 This idea was formalized by Tresillian and others in relation to human's ability to extrapolate an object's

268 location behind an occluder ${ }^{55,56}$. This is fundamentally different from what we propose; our findings

269 indicate that humans actively integrate information about temporal contexts and events even when the

270 object is visible. In other words, timing seem to be an integral component of how we interact with

271 dynamics stimuli, both to better estimate where they are (when they are visible), and to infer where they

272 might be (when they are occluded).

273 Our work does not address any potential role that timing information might play for the subsequent

274 sensorimotor adjustments after movement initiation. It is possible that knowledge about temporal cues

275 and contexts only inform movement initiation. This would indicate that temporal processing is only

276 engaged during the cognitive and/or motor planning stage of object interception. This is consistent with

277 numerous imaging and electrophysiological studies finding an important role for premotor and

278 supplementary motor areas in timing ${ }^{57-61}$. Alternatively, knowledge about movement durations may also

279 be used during movements although some studies have suggested that humans do not use timing

280 information when they have access to movement related state-dependent information ${ }^{62,63}$. 
281 It is worthwhile considering why the role of time was not noted in prior research on object interception.

282 We think that answer has to do with the simplicity of behavioral task used in laboratory settings (but see

283 some studies using more naturalistic paradigms or done with virtual reality ${ }^{47,64}$ ). Most experiments have

284 not included rich spatiotemporal event and/or contexts, where temporal cues become relevant. However,

285 real world examples of object interception take place in the presence of temporal statistics, spatial

286 landmarks, and temporal events such as collisions and/or reflections, all of which make knowledge about

287 time highly informative. A notable observation in our experiment was that subjects' estimate of TTC was

288 more accurate in the identity temporal context, possibly due to lower sensorimotor noise ${ }^{54}$. This improved

289 sensitivity may be due to the fact that temporal identity creates a rhythmic structure between the relevant

290 time intervals. If so, we would expect stronger effects when temporal events create sounds as auditory

291 rhythms and/or integer ratios are constrained by strong internal priors ${ }^{65}$. For example, intercepting a

292 bouncing ball may greatly benefit from the bounce sound, especially when visual information is uncertain

293 (e.g., a dribbling a basketball without looking at the ball). These considerations highlight the need for

294 future research to move beyond simple behavioral tasks and examine object interception in more

295 naturalistic settings where the underlying dynamics are governed by richer spatiotemporal contexts. We

296 speculate that doing so will further substantiate the importance of temporal events and contexts in

297 processing dynamic stimuli. 


\section{Online Methods}

\section{Subjects}

298 All subjects provided informed consent for experimental procedures which were approved by the

299 Committee On the Use of Humans as Experimental Subjects at the Massachusetts Institute of

300 Technology. Seven adult subjects participated in Experiment 1. A different group of seven adult subjects

301 participated in Experiment 2. Another group of eight adult subjects participated in Experiment 3. All

302 subjects had normal or corrected-to-normal vision.

\section{Procedures}

303 Subjects sat in a dark, quiet room at a distance of approximately $50 \mathrm{~cm}$ from a display monitor with a

304 refresh rate of $60 \mathrm{~Hz}$ and a resolution of 1920 by 1200 on an Apple Macintosh platform. Experiments

305 were controlled by an open-source software (MWorks; http://mworks-project.org/). All stimuli were

306 presented on a black background. Although eye movements were not monitored, all trials began with

307 central fixation spot that subjects were asked to hold their gaze on the fixation point throughout every

308 trial. Responses were made on a standard Apple Keyboard connected to the experimental machine.

309 We used three experiments to examine how people infer time-to-contact (TTC). Each experiment

310 consisted of conditions whose order was randomized across subjects. Each condition was tested twice in

311 two different days: the first session was used for training, and the second was used for the main test

312 session but the first 25 trials were considered as warm-up and were excluded from the main analysis.

313 Subjects were asked to press a key when the bar reached the target position. Feedback was provided to

314 indicate the actual bar position along the path when the key was pressed. The target position and the

315 stimulus feedback were shown in green when the produced time-to-contact (TTCp) was within an

316 experimentally defined window around the actual time-to-contact (TTCa), and red otherwise. To account 
317 for scalar variability, the window width was scaled with the actual time-to-contact (TTCa) with a constant

318 of proportionality, $k$. The value of $k$ was controlled by an adaptive one-up one-down procedure during

319 training condition, and eventually reached a stable value, $k_{0}$. We then set $k_{0}$ as feedback accuracy window

320 in the main test session.

\section{Experiment 1}

321 The objective of this experiment was to test whether subjects could improve their estimate of TTC by

322 integrating motion and timing cues. The trials were structured as follows: subject pressed a key to initiate

323 a trial. After a variable delay drawn randomly from a truncated exponential distribution (0.3-0.6 sec), a

324 bar started moving horizontally from a starting point along a 16-degrees long linear path toward a target

325 position at the end of the path (Fig. 1a). In each trial, the speed (v) of the bar was drawn from a discrete

326 uniform distribution (Fig. 1b).

327 The experiment consisted of three different conditions in terms of the information subjects were provided

328 with: one with motion cue, one with timing cue, and one with both (Fig. 2a). In the first condition, the

329 interception path consisted of two sections: a section where the stimulus movement was visible and a

330 section where it was occluded. The target was placed at the end of the occluded section. We denote this

331 condition by $\mathrm{I}(\mathrm{VM})$ as a shorthand for Interception in the presence of Visible Motion. In the second

332 condition, the motion was invisible throughout the path but the position of the stimulus was flashed at the

333 beginning of the path and when it reached the central fixation point in the middle of the path. We denote

334 this condition by $\mathrm{I}(\mathrm{FP})$ as a shorthand for Interception in the presence of Flashed Position. In the third

335 condition, the stimulus movement was visible early on, and additionally its position was flashed when it

336 reached the central fixation spot. Accordingly, we denote this condition by I(VM+FP). In tasks in which

337 the position was flashed, the flashes lasted $100 \mathrm{msec}$. The distribution of sample interval $\left(t_{1}\right)$ between the

338 start of the path and the time when the bar reached the fixation point was the same across the conditions. 
339 We also tested subjects in a cue conflict version of the $\mathrm{I}(\mathrm{VM}+\mathrm{FP})$ in which the flash at the central fixation

340 was jittered by -100 , 0 , or $100 \mathrm{msec}$ relative to when the actual stimulus reached the central

341 fixation (Supplementary Fig. 1a). These three jitter values were randomized and presented with equal

342 probability.

\section{Experiment 2}

343 The objective of this experiment was to test whether subjects could take advantage of temporal structure

344 in the absence of an explicit temporal cue to improve their performance. The task was similar to the

$345 \mathrm{I}(\mathrm{VM})$ condition in Experiment 1. A bar began to move from a starting point along a path. The bar was

346 initially visible and then disappeared behind an occluder. Subjects pressed a key when the bar reached the

347 target position at the end of the occluder. We tested subjects in three conditions (Fig. 3a). In all

348 conditions, the distance between the fixation spot and target $\left(\mathrm{d}_{2}\right)$ was set at 8 degrees while the visible

349 length between the starting and the fixation spots $\left(\mathrm{d}_{1}\right)$ was varied between 12,8 , and 5 degrees. We

350 expressed these conditions in terms of the ratio of $d_{2}$ over $d_{1}$, which we define as a gain factor $(G)$. The

351 corresponding $\mathrm{G}$ for the three conditions were 0.667 , 1, or 1.6. We recruited a new set of subjects for this

352 experiment to make sure that participants were not made sensitive to timing cues due to prior experience

353 with the I(FP) and/or I(VM+FP) tasks.

354 To evaluate the relative importance of speed and timing information, we also tested the newly recruited

355 subjects with the same gain factors but in the I(FP) condition (Fig. 3d). However, all I(FP) conditions

356 were tested after the subjects had completed the I(VM) conditions to avoid inadvertently sensitizing

357 subjects to timing cues. Overall, Experiment 2 consisted of 6 conditions in total.

\section{Experiment 3}

358 The objective of this experiment was to test whether the improved performance in Experiment 2 in

359 relation to the identity context was related to the distance identity or temporal identity. To facilitate the 
360 description of these conditions, let us define the sample interval $\left(t_{1}\right)$ as the interval associated with the

361 visible portion of the path, between the starting point and when the bar reached the central fixation $\left(d_{1}\right)$.

362 Similarly, we define the target interval $\left(\mathrm{t}_{2}\right)$ as the interval associated with the occluded part of the path

$363\left(\mathrm{~d}_{2}\right)$. Since the experiment involved changing the relative distances and/or durations, we additionally

364 define two a distance ratio that corresponds to the ration of the occluded length to the visible length $\left(\mathrm{G}_{\mathrm{d}}=\right.$

$\left.365 \mathrm{~d}_{2} / \mathrm{d}_{1}\right)$, and a duration ratio $\left(\mathrm{G}_{\mathrm{t}}=\mathrm{t}_{2} / \mathrm{t}_{1}\right)$ for the corresponding durations.

366 The experiment consisted of two variants of the $\mathrm{I}(\mathrm{VM})$ condition. In the first condition, we set $\mathrm{d}_{1}$ to 8

367 degrees and $d_{2}$ to 10 degrees $\left(G_{d}=1.25\right)$, and in the second condition both $d_{1}$ and $d_{2}$ were 10 degrees $\left(G_{d}\right.$

$368=1$ ). In the training sessions, similar to experiment 1 and 2 , the stimulus speed was constant throughout

369 the interception path. In the test sessions, unbeknownst to the subjects, immediately after the stimulus

370 entered the occluded segment, its speed was multiplied by 1.25 . This manipulation changed $\mathrm{G}_{\mathrm{t}}$ to 1 and

$3711 / 1.25$ in the first and second conditions respectively. In other words, in the first condition, $\mathrm{G}_{\mathrm{d}}=1.25$ and

$372 \mathrm{G}_{\mathrm{t}}=1$, whereas in the second condition $\mathrm{G}_{\mathrm{d}}=1$ and $\mathrm{G}_{\mathrm{t}}=1 / 1.25$. This manipulation allowed us to tease apart

373 the effect of distance and temporal identity contexts (Fig. 4a).

\section{Analysis}

374 We defined the actual time-to-contact $(T T C a)$ as the interval between when the bar passed the central

375 fixation to when it reached the target position. The produced time-to-contact (TTCP) was defined as the

376 time from when the bar passed the central fixation to when the subject pressed a key. Subjects that were

377 not sensitive to the range of sample intervals during the training session or had unstable performance were

378 excluded from the study. We considered a subject insensitive if the corresponding TTC $p$ distribution for

379 the longest TTCa was not significantly different from TTCp distribution for the shortest TTCa (paired t-

380 test at $\mathrm{p}=0.05$ level). Performance was considered unstable if the first and second order statistics of TTCp

381 were different between the first and second halves of the session (paired t-test at $\mathrm{p}=0.05$ level). 


\section{Summary statistics}

382 Following Jazayeri and Shadlen (2010), we characterized each subject's performance by computing the

383 following summary statistics for TTCp:

$$
\begin{aligned}
& \mathrm{BIAS}^{2}=\frac{1}{5} \times \sum_{i=1}^{5} \mathrm{BIAS}_{i}^{2}=\frac{1}{5} \times \sum_{i=1}^{5}\left(\overline{T T C}_{i}-T T C a_{i}\right)^{2} \\
& \mathrm{VAR}=\frac{1}{5} \times \sum_{i=1}^{5} \mathrm{VAR}_{i}=\frac{1}{5} \times \sum_{i=1}^{5}\left(\frac{1}{N_{i}-1} \sum_{k=1}^{N_{i}}\left(T T C p_{i k}-\overline{T T C p}_{i}\right)^{2}\right) \\
& \mathrm{RMSE}=\sqrt{\mathrm{BIAS}^{2}+\mathrm{VAR}}
\end{aligned}
$$

384 BIAS and VAR represent the average deviations and average variance over the five intervals included in

385 the prior distribution. $\mathrm{BIAS}_{\mathrm{i}}$ and $\mathrm{VAR}_{\mathrm{i}}$ represent the mean deviation and variance of produced times

$386(T T C p)$ for the $i$-th actual interval $(T T C a)$ with $N_{i}$ trials. It follows naturally that the overall root mean

387 squared error (RMSE) is equal to the square root of the sum of BIAS ${ }^{2}$ and VAR. To estimate the mean

388 and variance of summary statistics for individual subject in each condition, we resampled data with

389 replacement and repeated this resampling 100 times

\section{Effect size}

390 It is known that a relatively large sample size could lead to a smaller $\mathrm{p}$ value. Since there were more than

391100 trials in each session for each subject, we also measured the strength of difference between conditions

392 for each subject. We used Hedges' $g^{66}$, which is a measure to correct the bias in Cohen's $d$ to quantify the

393 distance between two distribution means. $g=0.2$ means small effect size, 0.5 means medium effect size,

394 and 0.8 means large effect size.

$g=J\left(n_{1}+n_{2}-2\right) \cdot \frac{\left(\bar{x}_{1}-\bar{x}_{2}\right)}{s *}$

395 where

$$
s *=\frac{\sqrt{\left(n_{1}-1\right) \cdot s_{1}^{2}+\left(n_{2}-1\right) \cdot s_{2}^{2}}}{n_{1}+n_{2}-2} \quad J(a)=\frac{\Gamma\left(\frac{a}{2}\right)}{\sqrt{\frac{a}{2}} \cdot \Gamma\left(\frac{a-1}{2}\right)}
$$




\section{The Bayesian observer model}

396 We developed a Bayesian observer model (Fig. 5a) based on previous work on interval reproduction ${ }^{24}$.

397 We modeled the prior distribution over sample intervals $\left(t_{l}\right)$ based on the ratio of the visible distance $\left(d_{l}\right)$

398 to the bar's speed (v). To simplify derivations, we modeled the discrete prior distributions used in the

399 experiment as a continuous uniform distribution ranging from the shortest to longest sample interval tests.

400 The shortest and longest intervals were computed in terms of the smallest and largest speeds $\left(v^{\min }\right.$ and $\left.401 v^{\max }\right)$.

$p\left(t_{1}\right)= \begin{cases}\frac{v^{\min } \cdot v^{\max }}{d_{1} \cdot\left(v^{\max }-v^{\min }\right)} & \frac{d_{1}}{v^{\max }} \leq t_{1} \leq \frac{d_{1}}{v^{\min }} \\ 0 & \text { otherwise }\end{cases}$

402 We assumed that subjects made two conditionally independent measurements when the bar was visible,

403 one associated with the speed of the bar, and another associated with duration of the visible period.

404 Following previous work on sensory measurements of time and speed ${ }^{26,33-36}$, we assumed that both

405 measurements were perturbed by scalar Gaussian noise. Specifically, we assumed that the standard

406 deviation of measured speed $\left(v_{m}\right)$ scales with speed $(v)$ with constant of proportionality $w_{m}$, and that the

407 standard deviation of measured elapsed time $\left(t_{m}\right)$ scales with elapsed time $\left(t_{l}\right)$ with constant of

408 proportionality $w_{m T}$. The variables $w_{m V}$ and $w_{m T}$ represent the Weber fraction for measurement of speed

409 and time, respectively. From the perspective of the observer who makes a measurement $t_{m}$ and $v_{m}$, but

410 does not know $t_{l}$ and $v$, the problem can be written in terms of the corresponding likelihood functions

$411 \lambda\left(t_{m} \mid t_{l}\right)$ and $\lambda\left(v_{m} \mid v\right)$ :

$$
\begin{aligned}
& \lambda\left(t_{m} \mid t_{1}\right)=\frac{1}{\sqrt{2 \pi \cdot\left(w_{m T} \cdot t_{1}\right)^{2}}} \times e^{\frac{-\left(t_{m}-t_{1}\right)^{2}}{2 \cdot\left(w_{m T} \cdot t_{1}\right)^{2}}} \\
& \lambda\left(v_{m} \mid v\right)=\frac{1}{\sqrt{2 \pi \cdot\left(w_{m V} \cdot v\right)^{2}}} \times e^{\frac{-\left(v_{m}-v\right)^{2}}{2 \cdot\left(w_{m V} \cdot v\right)^{2}}}
\end{aligned}
$$


412 To be able to combine the two likelihoods, we rewrote the likelihood associated with speed in terms of

413 distance and sample interval, as follows:

$$
\lambda\left(v_{m} \mid t_{1}\right)=\frac{1}{\sqrt{2 \pi \cdot\left(w_{m V} \cdot \frac{d_{1}}{t_{1}}\right)^{2}}} \times e^{\frac{-\left(v_{m}-\frac{d_{1}}{t_{1}}\right)^{2}}{2 \cdot\left(w_{m V} \cdot \frac{d_{1}}{t_{1}}\right)^{2}}}
$$

414 Assuming that the two measurements were conditionally independent, the posterior would be

$$
\pi\left(t_{1} \mid t_{m}, v_{m}\right)=\frac{p\left(t_{1}\right) \cdot p\left(t_{m} \mid t_{1}\right) \cdot p\left(v_{m} \mid t_{1}\right)}{\int p\left(t_{1}\right) \cdot p\left(t_{m} \mid t_{1}\right) \cdot p\left(v_{m} \mid t_{1}\right) d t_{1}}
$$

415 Following previous work ${ }^{24}$, we assumed that subjects' minimized expected loss using a quadratic loss 416 function, and modeled the inferred duration of the visible period based on the Bayes least-squares (BLS) 417 estimator (i.e., mean of the posterior). We assumed that the estimate was multiplied with a lossless gain 418 (G) to get time-to-contact compute (TTCe).

$$
\begin{aligned}
T T C_{e} & =G \cdot f_{\mathrm{BLS}}\left(t_{m}, v_{m}\right) \\
& =G \cdot \underset{t_{e}}{\operatorname{argmax}}\left[\int\left(t_{e}-t_{1}\right)^{2} \cdot \pi\left(t_{1} \mid t_{m}, v_{m}\right) d t_{1}\right] \\
& =G \cdot \int t_{1} \cdot \pi\left(t_{1} \mid t_{m}, v_{m}\right) d t_{1}
\end{aligned}
$$

419 For a uniform prior for sample interval $\left(\mathrm{t}_{1}\right)$, the time-to-contact estimate (TTCe) would be

$$
T T C_{e}=G \cdot \frac{\int_{t_{1, \min }}^{t_{1, \max }} t_{1} \cdot p\left(t_{m} \mid t_{1}\right) \cdot p\left(v_{m} \mid t_{1}\right) d t_{1}}{\int_{t_{1, \min }}^{t_{1, \max }} p\left(t_{m} \mid t_{1}\right) \cdot p\left(v_{m} \mid t_{1}\right) d t_{1}}
$$

420 The model was augmented by post-estimation noise to account for motor variability in the produced time-

421 to-contact $(T T C p)$. Following previous work $^{24,33,34,67}$, we assumed that the standard deviation of motor

422 noise was proportional to TTCe, with constant of proportionality of $w_{p}$ (Weber fraction for production).

423 We included an offset term $\left(b_{0}\right)$ in the fitting procedure to account for idiosyncratic stimulus- and prior424 independent biases observed in responses. 
$p\left(T T C_{p} \mid T T C_{e}\right)=\frac{1}{\sqrt{2 \pi \cdot\left(w_{p} \cdot T T C_{e}\right)^{2}}} \times e^{\frac{-\left(T T C_{p}-\left(T T C_{e}+b_{0}\right)\right)^{2}}{2 \cdot\left(w_{p} \cdot\left(T T C_{e}+b_{0}\right)\right)^{2}}}$

425 Using chain rule and marginalization of hidden variables, we wrote the the conditional probability of

426 produced time-to-contact (TTCp) for a each actual time-to-contact (TTCa) as follows:

$$
\begin{aligned}
& p\left(T T C_{p} \mid T T C_{a} ; w_{m T}, w_{m V}, w_{p}, b_{0}\right) \\
& =\iint p\left(T T C p \mid G_{d} \cdot f_{\mathrm{BLS}}\left(t_{m}, v_{m}\right) ; w_{p}, b_{0}\right) \cdot p\left(t_{m} \mid\left(T T C_{a} / G_{d}\right) ; w_{m T}\right) \cdot p\left(v_{m} \mid\left(T T C_{a} / G_{d}\right) ; w_{m V}\right) d t_{m} d v_{m}
\end{aligned}
$$

\section{Fitting procedure}

427 For fitting procedure, we assumed that $T T C p$ values associated with any TTCa were independent across

428 trials and thus expressed the joint conditional probability of individual TTCP values across all the $\mathrm{N}$ trials

429 by the product of their individual conditional probabilities.

$\log p\left(T T C_{p}^{1}, T T C_{p}^{2}, \ldots, T T C_{p}^{N} \mid T T C_{a} ; w_{m V}, w_{m T}, w_{p}, b_{0}\right)=\sum_{i=1}^{N} \log p\left(T T C_{p}^{i} \mid T T C_{a} ; w_{m V}, w_{m T}, w_{p}, b_{0}\right)$

430 We used fminsearch algorithm to find the model parameters that maximized the likelihood of model

431 parameters across all $T T C a$ and $T T C p$ values measured psychophysically. Integrals were approximated

432 numerically using the global adaptive quadrature ${ }^{68}$. We repeated the search with different initial values 10

433 times, and verified that the likelihood functions were stable with respect to initial values.

\section{Predicting behavior in temporal identity context}

434 Instead of fitting the Bayesian model to each dataset, we asked whether we could fit the model to some

435 conditions and then use parameters of the fitted model to predict behavior in other conditions. We aimed

436 to predict behavior in the most important condition where subjects integrated speed with the identity

437 temporal context; i.e., $\mathrm{I}(\mathrm{VM})$ with $\mathrm{G}=1$. We assumed that the noise associated with the measurement of

$438 \mathrm{t}_{1}$ is the same in the I(VM) and I(FP) tasks and therefore, used the Bayesian model to the I(FP) task for G

$439=1$ to estimate $\mathrm{w}_{\mathrm{mT}}$ (Fig. 5b, left). We further assumed that the measurement of speed in I(VM) task 
440 would be the same across two different gains $(G=1$ and $G=0.667)$, given that the accuracy of speed

441 measurement saturated rapidly (Supplementary Fig. 2). We first found $\mathrm{w}_{\mathrm{mT}}$ for $\mathrm{G}=0.667$ from I(FP)

442 task, and then used this value to fit a Bayesian model to $\mathrm{I}(\mathrm{VM})$ task with $\mathrm{G}=0.667$ to estimate $\mathrm{w}_{\mathrm{mV}}$ (Fig.

$443 \mathbf{5 b}$, right). Finally, we used the $\mathrm{w}_{\mathrm{mT}}$ inferred from $\mathrm{I}(\mathrm{FP})$ with $\mathrm{G}=1$ and $\mathrm{w}_{\mathrm{mV}}$ inferred from $\mathrm{I}(\mathrm{VM})$ with $\mathrm{G}$

$444=0.667$ to predict behavior in the $\mathrm{I}(\mathrm{VM})$ task with $\mathrm{G}=1$ in (Fig. 5c). 


\section{References}

1. Bootsma, R. J. \& Oudejans, R. R. Visual information about time-to-collision between two objects. J. Exp. Psychol. Hum. Percept. Perform. 19, 1041-1052 (1993).

2. Lee, D. N., Young, D. S., Reddish, P. E., Lough, S. \& Clayton, T. M. Visual timing in hitting an accelerating ball. Q. J. Exp. Psychol. A 35, 333-346 (1983).

3. Savelsbergh, G. J., Whiting, H. T. \& Bootsma, R. J. Grasping tau. J. Exp. Psychol. Hum. Percept. Perform. 17, 315$322(1991)$.

4. Smeets, J. B., Brenner, E., Trébuchet, S. \& Mestre, D. R. Is judging time-to-contact based on 'tau'? Perception 25, 583-590 (1996).

5. Brenner, E. \& Smeets, J. Intercepting moving objects: do eye-movements matter. Space and time in perception and action (2010).

6. Kwon, O.-S. \& Knill, D. C. The brain uses adaptive internal models of scene statistics for sensorimotor estimation and planning. Proceedings of the National Academy of Sciences 110, E1064-E1073 (2013).

7. Mrotek, L. A., Flanders, M. \& Soechting, J. F. Interception of targets using brief directional cues. Exp. Brain Res. 156, 94-103 (2004).

8. Mrotek, L. A. \& Soechting, J. F. Target interception: hand-eye coordination and strategies. J. Neurosci. 27, 72977309 (2007).

9. Zago, M., McIntyre, J., Senot, P. \& Lacquaniti, F. Visuo-motor coordination and internal models for object interception. Exp. Brain Res. 192, 571-604 (2009).

10. Bosco, G., Delle Monache, S. \& Lacquaniti, F. Catching what we can't see: manual interception of occluded fly-ball trajectories. PLoS One 7, e49381 (2012).

11. Brouwer, A.-M., Brenner, E. \& Smeets, J. B. J. Hitting moving objects: is target speed used in guiding the hand? Exp. Brain Res. 143, 198-211 (2002).

12. Soechting, J. F., Juveli, J. Z. \& Rao, H. M. Models for the extrapolation of target motion for manual interception. J. Neurophysiol. 102, 1491-1502 (2009).

13. McIntyre, J., Zago, M., Berthoz, A. \& Lacquaniti, F. Does the brain model Newton's laws? Nat. Neurosci. 4, 693694 (2001). 
14. Faisal, A. A., Selen, L. P. J. \& Wolpert, D. M. Noise in the nervous system. Nat. Rev. Neurosci. 9, 292-303 (2008).

15. Ahrens, M. B. \& Sahani, M. Observers exploit stochastic models of sensory change to help judge the passage of time. Curr. Biol. 21, 200-206 (2011).

16. Brenner, E. \& Smeets, J. B. J. Sources of variability in interceptive movements. Exp. Brain Res. 195, 117-133 (2009).

17. Pellizzer, G. \& Hedges, J. H. Motor planning: effect of directional uncertainty with continuous spatial cues. Exp. Brain Res. 154, 121-126 (2003).

18. Scheidt, R. A., Dingwell, J. B. \& Mussa-Ivaldi, F. A. Learning to move amid uncertainty. J. Neurophysiol. 86, 971$985(2001)$.

19. Ota, K., Shinya, M. \& Kudo, K. Motor planning under temporal uncertainty is suboptimal when the gain function is asymmetric. Front. Comput. Neurosci. 9, 88 (2015).

20. van Beers, R. J., Baraduc, P. \& Wolpert, D. M. Role of uncertainty in sensorimotor control. Philos. Trans. R. Soc. Lond. B Biol. Sci. 357, 1137-1145 (2002).

21. Miyazaki, M., Nozaki, D. \& Nakajima, Y. Testing Bayesian models of human coincidence timing. J. Neurophysiol. 94, 395-399 (2005).

22. Aschersleben, G. \& Prinz, W. Synchronizing actions with events: the role of sensory information. Percept. Psychophys. 57, 305-317 (1995).

23. Chen, Y., Ding, M. \& Kelso, J. Long memory processes (1/f $\alpha$ type) in human coordination. Phys. Rev. Lett. (1997).

24. Jazayeri, M. \& Shadlen, M. N. Temporal context calibrates interval timing. Nat. Neurosci. 13, 1020-1026 (2010).

25. Knill, D. C. \& Pouget, A. The Bayesian brain: the role of uncertainty in neural coding and computation. Trends Neurosci. 27, 712-719 (2004).

26. Stocker, A. A. \& Simoncelli, E. P. Noise characteristics and prior expectations in human visual speed perception. Nat. Neurosci. 9, 578-585 (2006).

27. Körding, K. P., Ku, S.-P. \& Wolpert, D. M. Bayesian integration in force estimation. J. Neurophysiol. 92, 31613165 (2004).

28. Körding, K. P. \& Wolpert, D. M. Bayesian integration in sensorimotor learning. Nature 427, $244-247$ (2004).

29. Ernst, M. O. \& Banks, M. S. Humans integrate visual and haptic information in a statistically optimal fashion. Nature 415, 429-433 (2002). 
30. Tassinari, H., Hudson, T. E. \& Landy, M. S. Combining Priors and Noisy Visual Cues in a Rapid Pointing Task. J. Neurosci. 26, 10154-10163 (2006).

31. Hillis, J. M., Ernst, M. O., Banks, M. S. \& Landy, M. S. Combining sensory information: mandatory fusion within, but not between, senses. Science 298, 1627-1630 (2002).

32. Gray, R. \& Regan, D. Accuracy of estimating time to collision using binocular and monocular information. Vision Res. 38, 499-512 (1998).

33. Gibbon, J., Church, R. M. \& Meck, W. H. Scalar Timing in Memory. Ann. N. Y. Acad. Sci. 423, 52-77 (1984).

34. Rakitin, B. C. et al. Scalar expectancy theory and peak-interval timing in humans. J. Exp. Psychol. Anim. Behav. Process. 24, 15-33 (1998).

35. Welch, L. The perception of moving plaids reveals two motion-processing stages. Nature 337, 734-736 (1989).

36. McKee, S. P., Silverman, G. H. \& Nakayama, K. Precise velocity discrimination despite random variations in temporal frequency and contrast. Vision Res. 26, 609-619 (1986).

37. Jazayeri, M. \& Shadlen, M. N. A Neural Mechanism for Sensing and Reproducing a Time Interval. Curr. Biol. 25, 2599-2609 (2015).

38. Acerbi, L., Wolpert, D. M. \& Vijayakumar, S. Internal representations of temporal statistics and feedback calibrate motor-sensory interval timing. PLoS Comput. Biol. 8, e1002771 (2012).

39. Cicchini, G. M., Arrighi, R., Cecchetti, L., Giusti, M. \& Burr, D. C. Optimal encoding of interval timing in expert percussionists. J. Neurosci. 32, 1056-1060 (2012).

40. Tresilian, J. R. Visually timed action: time-out for 'tau'? Trends Cogn. Sci. 3, 301-310 (1999).

41. Hecht, H. \& Savelsbergh, G. J. P. in Advances in Psychology (ed. Heiko Hecht and Geert J.P. Savelsburgh) Volume 135, 1-11 (North-Holland, 2004).

42. López-Moliner, J., Field, D. T. \& Wann, J. P. Interceptive timing: prior knowledge matters. J. Vis. 7, 11.1-8 (2007).

43. Brenner, E. \& Smeets, J. B. J. How people achieve their amazing temporal precision in interception. J. Vis. 15, (2015).

44. Lacquaniti, F. \& Maioli, C. The role of preparation in tuning anticipatory and reflex responses during catching. $J$. Neurosci. 9, 134-148 (1989).

45. Hecht, H., Kaiser, M. K. \& Banks, M. S. Gravitational acceleration as a cue for absolute size and distance? Percept. Psychophys. 58, 1066-1075 (1996). 
46. Lee, D. D., Ortega, P. A. \& Stocker, A. A. Dynamic belief state representations. Curr. Opin. Neurobiol. 25, 221-227 (2014).

47. Fooken, J., Yeo, S.-H., Pai, D. K. \& Spering, M. Eye movement accuracy determines natural interception strategies. J. Vis. 16, 1 (2016).

48. Dubrowski, A., Lam, J. \& Carnahan, H. Target velocity effects on manual interception kinematics. Acta Psychol. 104, 103-118(2000).

49. Resulaj, A., Kiani, R., Wolpert, D. M. \& Shadlen, M. N. Changes of mind in decision-making. Nature 461, 263-266 (2009).

50. Jacobs, R. A. \& Fine, I. Experience-dependent integration of texture and motion cues to depth. Vision Res. 39, 4062-4075 (1999).

51. Cavallo, V. \& Laurent, M. Visual information and skill level in time-to-collision estimation. Perception 17, 623-632 (1988).

52. Mason, A. H. \& Carnahan, H. Target viewing time and velocity effects on prehension. Exp. Brain Res. 127, 83-94 (1999).

53. Krukowski, A. E., Pirog, K. A., Beutter, B. R., Brooks, K. R. \& Stone, L. S. Human discrimination of visual direction of motion with and without smooth pursuit eye movements. J. Vis. 3, 831-840 (2003).

54. Remington, E. \& Jazayeri, M. Late Bayesian inference in sensorimotor behavior. bioRxiv 130062 (2017). doi:10.1101/130062

55. Tresilian, J. R. Perceptual and cognitive processes in time-to-contact estimation: analysis of prediction-motion and relative judgment tasks. Percept. Psychophys. 57, 231-245 (1995).

56. DeLucia, P. R. \& Liddell, G. W. Cognitive motion extrapolation and cognitive clocking in prediction motion task. J. Exp. Psychol. Hum. Percept. Perform. 24, 901-914 (1998).

57. Merchant, H., Pérez, O., Zarco, W. \& Gámez, J. Interval tuning in the primate medial premotor cortex as a general timing mechanism. J. Neurosci. 33, 9082-9096 (2013).

58. Harrington, D. L., Zimbelman, J. L., Hinton, S. C. \& Rao, S. M. Neural modulation of temporal encoding, maintenance, and decision processes. Cereb. Cortex 20, 1274-1285 (2010).

59. Mita, A., Mushiake, H., Shima, K., Matsuzaka, Y. \& Tanji, J. Interval time coding by neurons in the presupplementary and supplementary motor areas. Nat. Neurosci. 12, 502-507 (2009). 
60. Kunimatsu, J. \& Tanaka, M. Alteration of the timing of self-initiated but not reactive saccades by electrical stimulation in the supplementary eye field. Eur. J. Neurosci. 36, 3258-3268 (2012).

61. Cui, X., Stetson, C., Montague, P. R. \& Eagleman, D. M. Ready...go: Amplitude of the FMRI signal encodes expectation of cue arrival time. PLoS Biol. 7, e1000167 (2009).

62. Diedrichsen, J., Criscimagna-Hemminger, S. E. \& Shadmehr, R. Dissociating timing and coordination as functions of the cerebellum. J. Neurosci. 27, 6291-6301 (2007).

63. Conditt, M. A. \& Mussa-Ivaldi, F. A. Central representation of time during motor learning. Proc. Natl. Acad. Sci. U. S. A. 96, 11625-11630 (1999).

64. Diaz, G., Cooper, J., Rothkopf, C. \& Hayhoe, M. Saccades to future ball location reveal memory-based prediction in a virtual-reality interception task. J. Vis. 13, (2013).

65. Jacoby, N. \& McDermott, J. H. Integer Ratio Priors on Musical Rhythm Revealed Cross-culturally by Iterated Reproduction. Curr. Biol. 27, 359-370 (2017).

66. Hedges, L. V. Statistical Methods for Meta-Analysis. (Academic Press, 1985).

67. Gallistel, C. R. \& Gibbon, J. Time, rate, and conditioning. Psychol. Rev. 107, 289-344 (2000).

68. Shampine, L. F. Vectorized adaptive quadrature in MATLAB. J. Comput. Appl. Math. 211, 131-140 (2008). 


\section{Box}

a
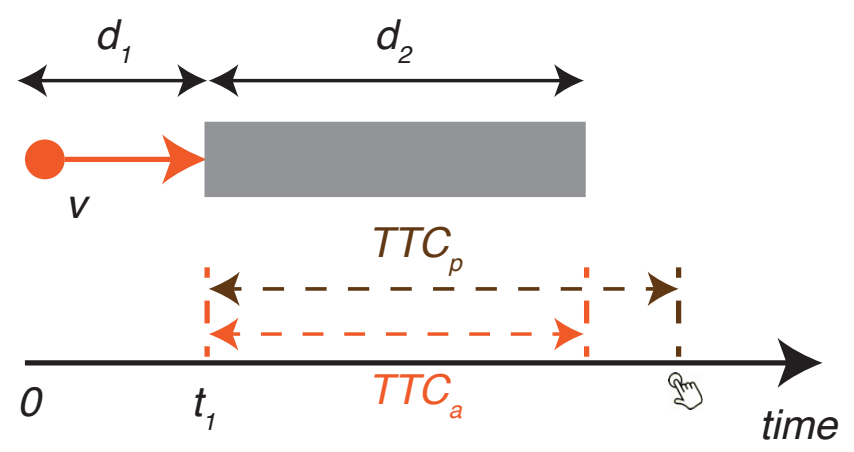

b

\section{stimulus measurement estimation}

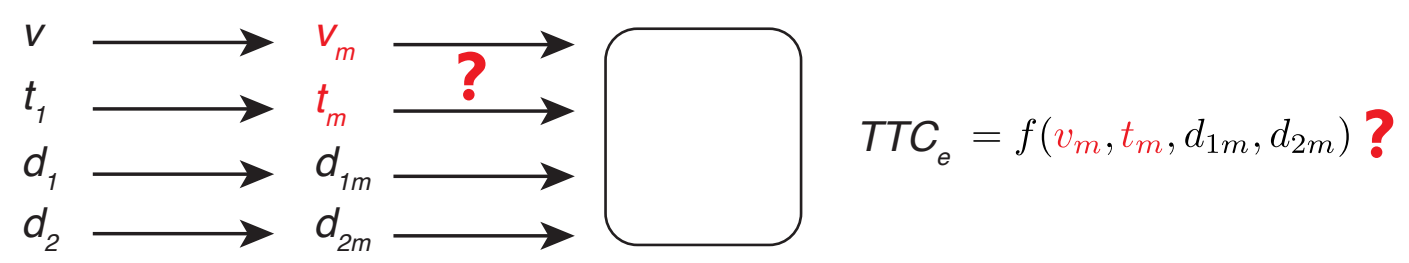

Box 1. Schematic paradigm and hypothesis for object interception.

(a) The overall logic of the experimental design. Subjects are asked to press a key when an bar moving with speed, $v$, would arrive at a target. The movement path is divided into a first section where the bar is visible, and a second section where the bar is invisible (i.e., virtually occluded). The subscript 1 and 2 are used to denote the distance $(d)$, speed $(v)$ and duration $(t)$ of the two sections, respectively. Subjects' behavior is evaluated by comparing the actual time-to-contact $(T T C a)$ to the produced time-to-contact $(T T C p)$, both of which are measured with respect to the moment the bar goes behind the occluder. (b) An estimate of TTC (denoted TTCe) can be derived by applying an appropriate transformation (noted as function, $f$ ) to measured stimulus parameters $\left(v_{m}, t_{m}, d_{1 m}, d_{2 m}\right)$. The key question we focus on is whether subjects rely solely on the speed $\left(v_{m}\right)$, or they additionally incorporate temporal cues $\left(t_{m}\right)$. 


\section{Figures}

a

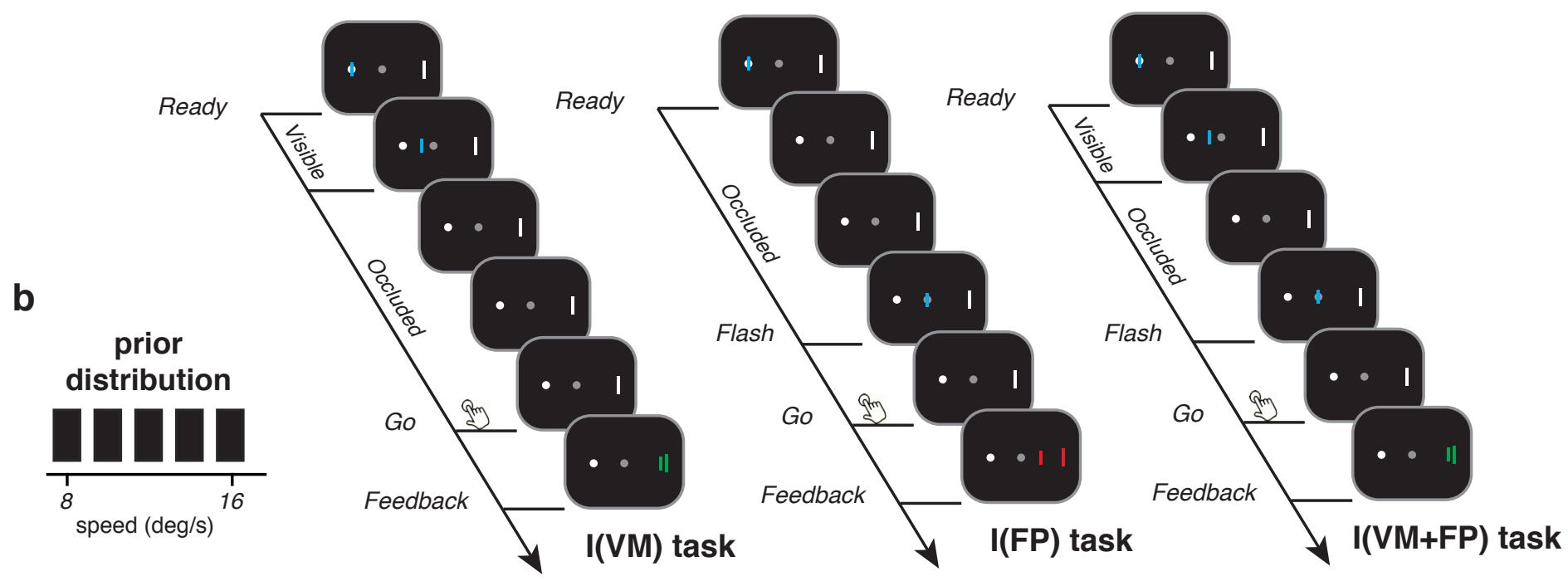

C

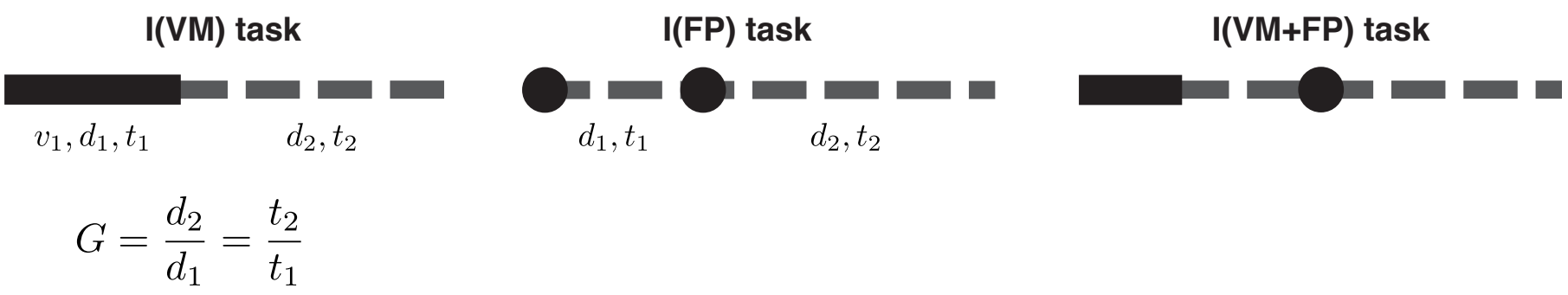

Figure 1. Experimental design and behavioral tasks.

(a) Behavioral tasks. A bar moved from an initial point to the left of the fixation point to a target point to the right of the fixation point. The initial and target points were present throughout the trial. Subjects had to press a key when they judged the moving bar to have arrived at the target. Using this basic design, we tested subjects behavior in three conditions. In the I(VM) task (Interception with Visual Motion), the stimulus movement was initially visible and then invisible as if behind an (imaginary) occluder. In the I(FP) task (Interception with Flashed Position), the motion was not displayed and the stimulus was only flashed at the starting point and when it reached the fixation point. In the I(VM+FP) task, both the initial movement and the intermediate flash at the fixation point were displayed. In all trials, we provided feedback by presenting the position of the stimulus at the time of keypress. To reinforce accuracy, the target position and the stimulus feedback were shown in green when TTCp was within an experimentally defined window around TTCa, and red otherwise (see Methods). (b) Prior distribution of the stimulus speed. Speed was sampled from a discrete uniform distribution from 8 to 16 degree per second, and varied across trials. (c) Simplified symbolic representation of I(VM), I(FP), and I(VM+FP) conditions. The circles correspond to times when the stimulus was flashed, the solid lines to when the motion was displayed, and the dashed lines to when the stimulus was occluded. We used this symbolic representation as key for other figures. 

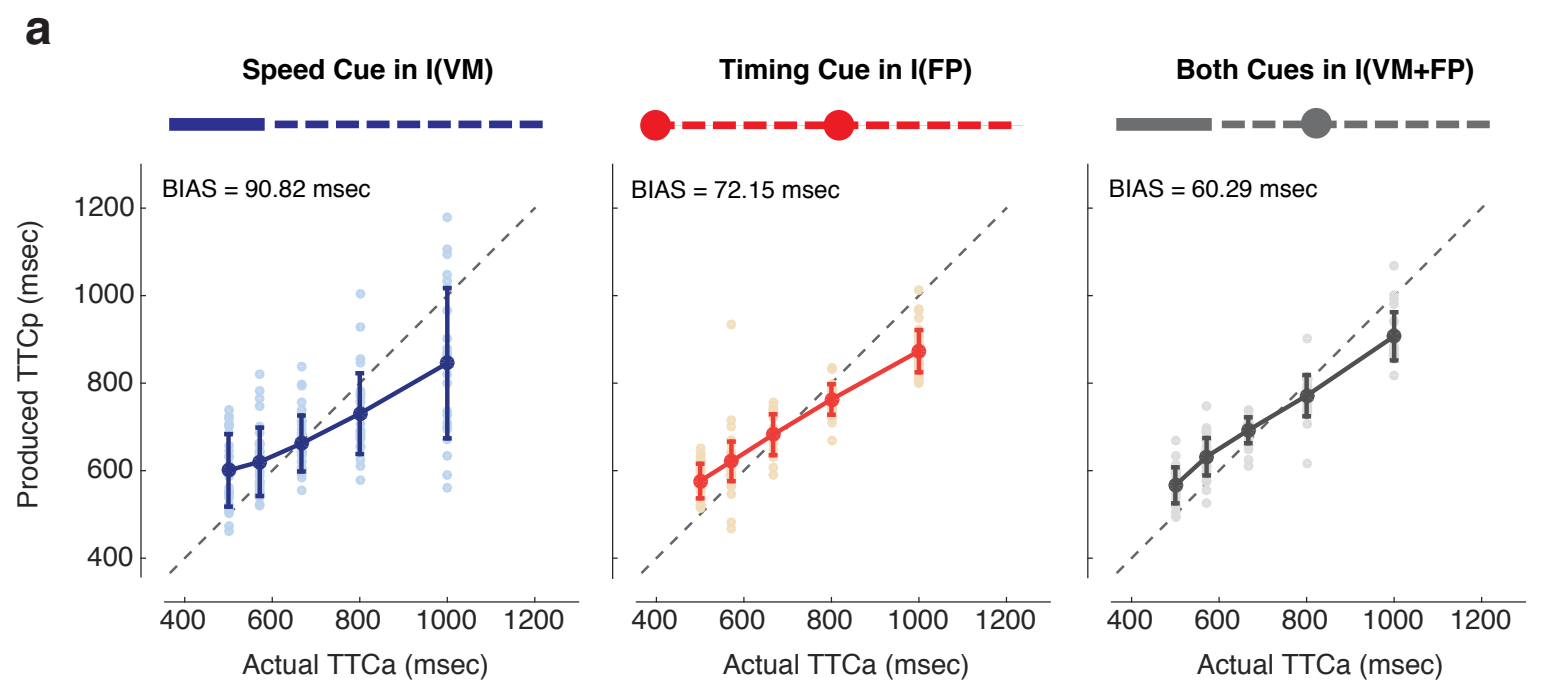

b

C
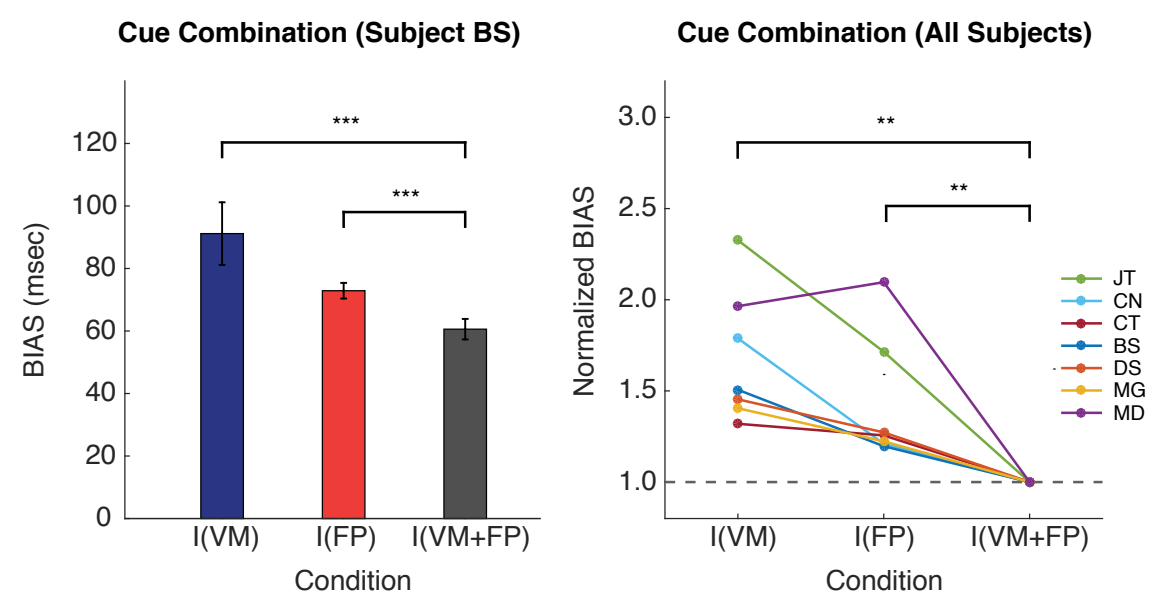

Figure 2. Interception using speed and explicit timing cues (Experiment 1).

(a) Behavior of a typical subject for different conditions in Experiment 1. The left panel corresponds to the I(VM) task were the speed of the stimulus was evident from the initial visible section of the movement. The middle panel corresponds to the I(FP) task in which the stimulus was flashed at the starting point and the central fixation point. The right panel corresponds to the $\mathrm{I}(\mathrm{VM}+\mathrm{FP})$ task where both the initial speed and the intermediate flash were presented. Performance was quantified by comparing subject's produced time-to-contact (TTCp) to the actual timeto-contact (TTCa). TTCa was defined as the time between when the bar reached the central fixation to when it reached the target. TTCp was defined as the time between when the bar reached the central fixation to when the button was pressed. Light dots and dark circles show TTCp in each trial and the corresponding averages for each $T T C a$. BIAS in each plot was quantified as the average error over the five distinct TTCa of the prior distribution, i.e., the root mean square of differences between five solid dark circle and the corresponding diagonal dash line on the plot. (b) BIAS comparison across conditions for a typical subject. We estimated the standard error through resampling data with 100 repetitions. BIAS was smaller for the I(FP) compared to I(VM), and smallest in the $\mathrm{I}(\mathrm{VM}+\mathrm{FP})$ condition. (c) Normalized BIAS across conditions for all subjects $(\mathrm{N}=7)$ shown in different colors. Normalized BIAS was obtained by dividing BIAS in all conditions with BIAS in the I(VM+FP) condition. Across subjects, BIAS patterns were similar to the typical subject in panel $\mathrm{b}$. 

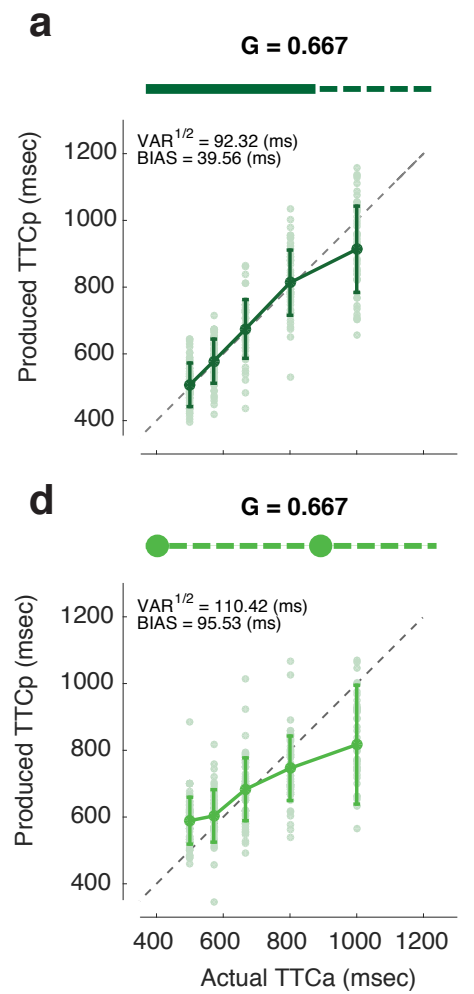
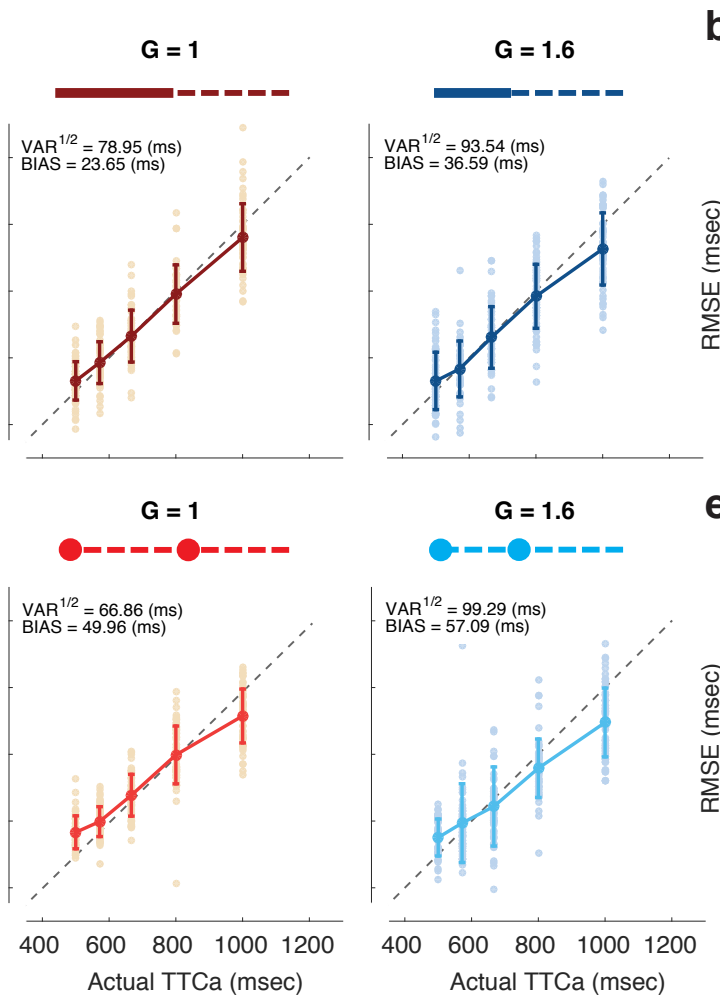

b
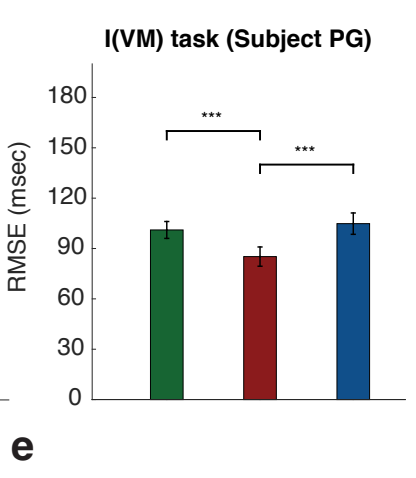

I(FP) task (Subject PG)

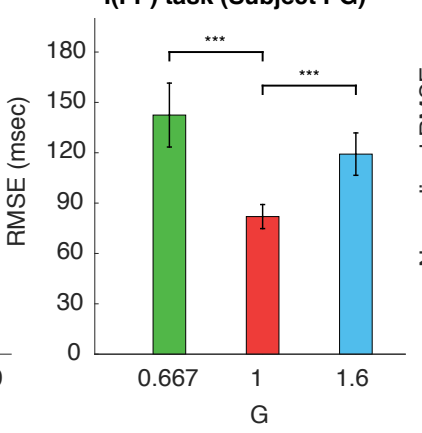

C

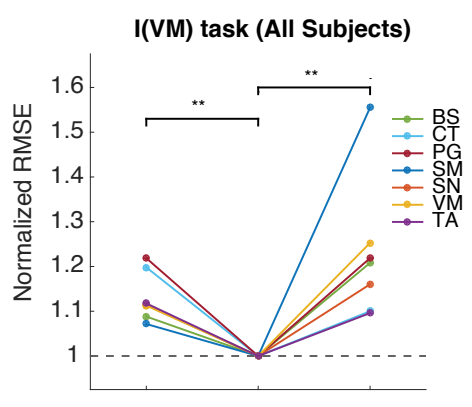

Figure 3. Interception using speed and implicit knowledge of temporal context (Experiment 2)

(a) Behavior of a typical subject in three variants of the I(VM) task with three different visible lengths and the same occluded length. Each condition was identified by a gain factor $(G)$ that quantified the ratio of the occluded to visible length. Since the bar moved at a constant speed throughout each trial, the gain also reflected the ratio of the duration of occluded and visible sections of the path. Performance was quantified by comparing subjects' produced time-to-contact (TTCP) to the actual time-to-contact (TTCa). Light dots and dark circles show TTCp in each trial and the corresponding averages for each TTCa. BIAS was defined as described in Figure 2. VAR is the average variance of $T T C p$ over the five intervals tested (TTCa) of the prior distribution. (b) Comparison of performance in terms of RMSE across conditions for a typical subject in I(VM) task. We estimated the standard error of RMSE through resampling data with 100 repetitions. (c) Normalized RMSE as a function of G for I(VM) task across all subjects $(N=7)$. RMSE in each condition was divided by RMSE when the gain was identical $(G=1)$. Different colors lines correspond to different subjects. (d) Behavior of a typical subject for I(FP) task with different gains (G) in Experiment 2. (e) Comparison across conditions for a typical subject in I(FP) task. (f) Normalized RMSE as a function of $\mathrm{G}$ for $\mathrm{I}(\mathrm{FP})$ task across all subjects $(\mathrm{N}=7)$. For all subjects and in both tasks, RMSE was smallest for $\mathrm{G}=1$ indicating best performance when the visible and occluded lengths were the same length (identity context, see main text). 
a

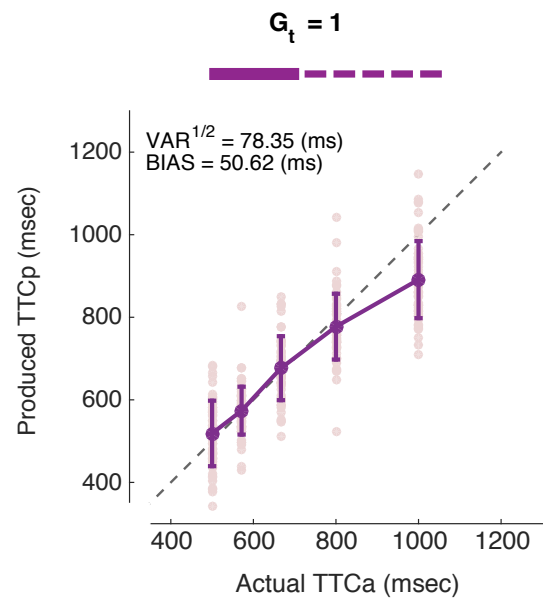

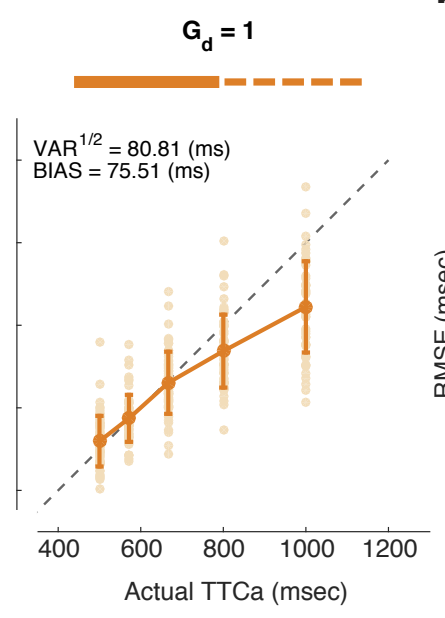

b

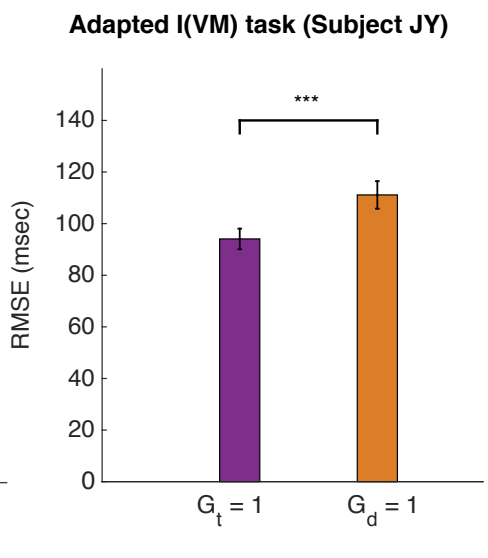

C

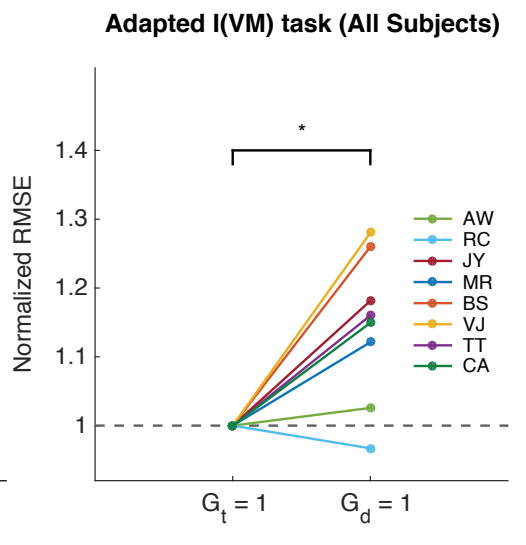

Figure 4. Interception using distance and temporal identity contexts (Experiment 3)

(a) Behavior of a typical subject for two variants of the $\mathrm{I}(\mathrm{VM})$ task, the temporal identity context $\left(G_{t}=1\right)$ and the distance identity context $\left(G_{d}=1\right)$. In both variants, unbeknownst to the subject, the speed behind the occluder was multiplied by 1.25 ( $25 \%$ faster than the visible section). $G_{t}=1$ : The durations of movement in the visible and occluded sections were the same. Because of speed difference between the two sections, the visible distance was shorter than the occluded distance. $G_{d}=1$ : The visible distance was same as the occluded distance, but the corresponding durations were different. (b) Comparison between the two conditions of I(VM) shown for a typical subject. We estimated the standard error of RMSE through resampling data with 100 repetitions. (c) Normalized RMSE across all subjects $(\mathrm{N}=8)$. Different colored lines represented different subjects. 


\section{time measurement}
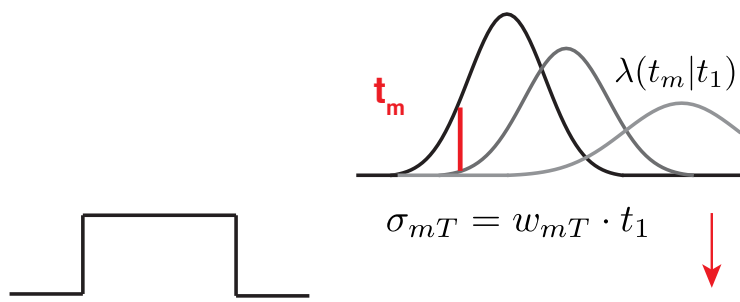

prior

$p\left(t_{1}\right)=\frac{d_{1}}{v}$

b

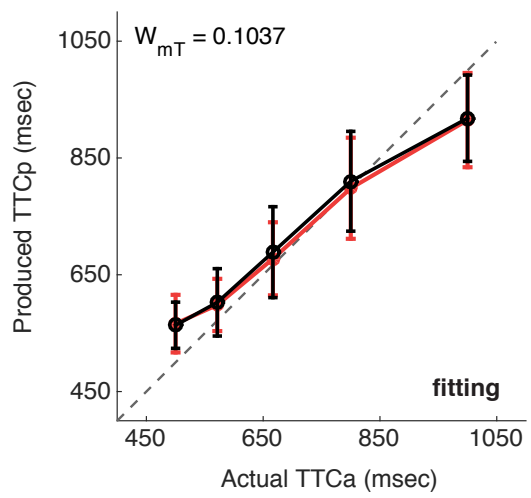

$\mathrm{W}_{\mathrm{m \tau}}$ estimation

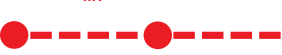

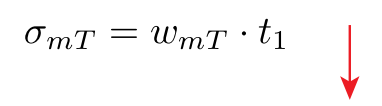

BLS estimation speed measurement

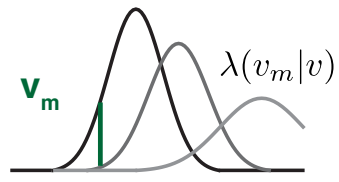

$\sigma_{m V}=w_{m V} \cdot v$

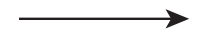

production

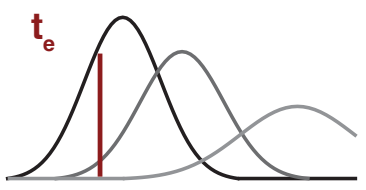

$T T C_{e}=G \cdot t_{e}=G \cdot f_{\mathrm{BLS}}\left(t_{m}, v_{m}\right)=G \cdot E\left[t_{1} \mid t_{m}, v_{m}\right]$

$\mathbf{W}_{\mathrm{mv}}$ estimation

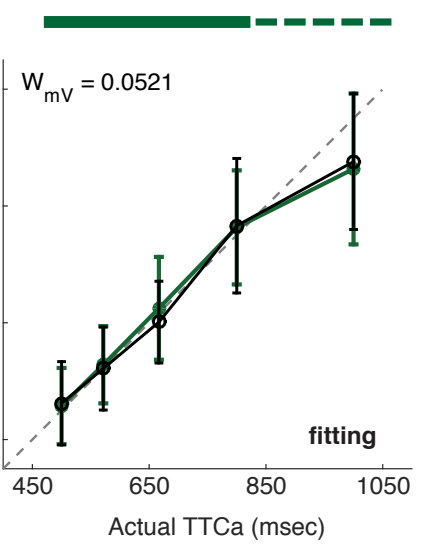

C

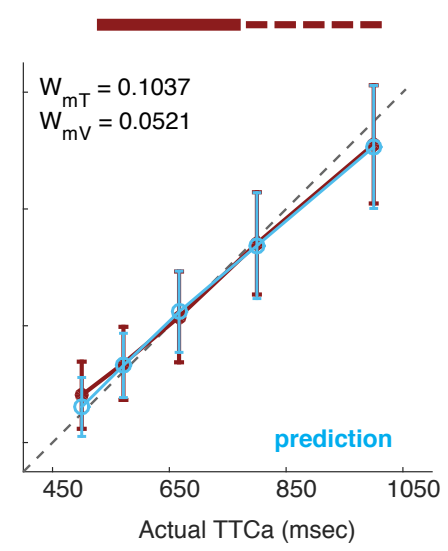

d

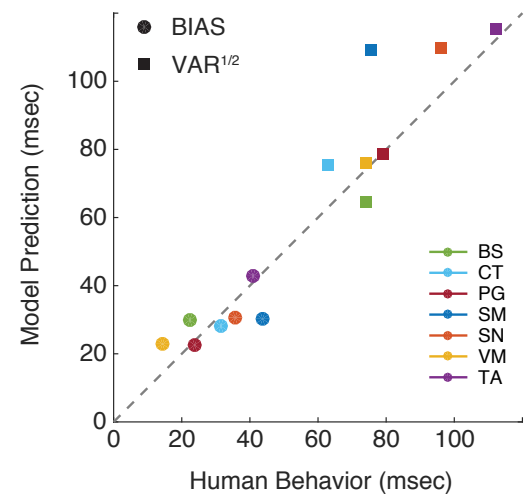

Figure 5. The Bayesian observer model of interception integrating speed and timing cues

(a) The Bayesian observer model for the I(VM) task. On each trial, the speed (v) was drawn from a uniform prior distribution. We used the relationship between the distance of the visible section $\left(d_{1}\right)$ and speed to express the prior in terms of the duration the bar is visible $\left(\mathrm{p}\left(t_{l}\right)\right.$; left). We assumed that the observer makes two conditionally independent measurements of $v$ and $t_{l}$, which we denoted by $v_{m}$ (red vertical line) and $t_{m}$ (green vertical line), respectively. We assumed that $v_{m}$ and $t_{m}$ are perturbed by zero-mean Gaussian noise with standard deviations $\left(\sigma_{m V}\right.$ and $\sigma_{m T}$ ) proportional to $v$ and $t_{l}$ (top Gaussian curves) with constant of proportionality of $w_{m V}$ and $w_{m T}$, respectively. The Bayesian observer computes the posterior from the likelihood functions, $\lambda\left(v_{m} \mid v\right)$ and $\lambda\left(t_{m} \mid t_{l}\right)$, and the prior, and uses a Bayes-Least-Squares (BLS) estimator, $f_{B L S}$, to infer the movement duration in the visible section, which we denoted by $t_{e}$ (brown vertical line) from $v_{m}$ and $t_{m}$. This estimate is then multiplied by the distance gain $(\mathrm{G})$ to obtain an optimal estimate of time-to-contact (TTCe). Finally, the model incorporates motor variability via additional noise in the production stage. We modeled this noise as a sample from a zero-mean Gaussian with standard deviation scaling with $T T C_{e}$ with scaling factor $w_{p}$. (b) The left panel ( $w_{m T}$ estimation) shows the behavior of a Bayesian observer model (red) fitted to the data (black) for a typical subject in I(FP) task with $G=1$. Since the movement of the bar in the $\mathrm{I}(\mathrm{FP})$ task is not visible, we estimated $\mathrm{w}_{\mathrm{mT}}$ from a Bayesian model that relies on the prior and $t_{m}$, but not $v_{m}$. The right panel ( $w_{m V}$ estimation) shows the Bayesian model (green lines) and the corresponding data (black) for the $\mathrm{I}(\mathrm{VM})$ task with $\mathrm{G}=0.667$. In the $\mathrm{I}(\mathrm{VM})$ condition, the observer has access to both speed and time. Therefore, we estimated $w_{m V}$ from a Bayesian model that uses the prior, $t_{m}$ and $v_{m}$ with $w_{m T}$ inferred from I(FP) with $\mathrm{G}=0.667$. (c) Behavior (black) and model prediction (blue) for a typical subject in the $\mathrm{I}(\mathrm{VM})$ task with $\mathrm{G}=1$. The prediction was made based on a Bayesian model whose $w_{m T}$ and $w_{m V}$ were derived in (b). (d) Comparison of summary statistics (BIAS and VAR ${ }^{1 / 2}$ ) between human behavior (abscissa) and predictions from a Bayesian model (ordinate) across subjects $\left(\mathrm{N}=7\right.$ ). BIAS and $\mathrm{VAR}^{1 / 2}$ of the model was computed based on averages of 100 simulations of the Bayesian observer model. Different colors correspond to different subjects 